\title{
Aripiprazole-induced Diplopia: A Rare Case Report
}

\author{
Manaswini Mullapudi ${ }^{1}, \mathrm{R}$ Madan $^{2}$, Vishnu Vardhan $^{3}$, M Vidhyavathi $^{4}$
}

\begin{abstract}
Background: Aripiprazole is a second-generation antipsychotic (SGA) drug. The commonly reported side effects of aripiprazole are headache, nausea, constipation, somnolence, or insomnia. However, ocular side effects are rarely reported with aripiprazole, with myopia being the most commonly reported. Eight cases of aripiprazole-induced myopia were reported and only two cases of diplopia secondary to aripiprazole use were reported in literature.

Aim: We aim to report a case of aripiprazole-induced diplopia in a 28-year-old woman who was treated for depression with psychotic symptoms. Case description: A 28-year-old woman who was a known case of depression with psychotic symptoms was treated with $3 \mathrm{mg}$ oral risperidone but had to be switched to aripiprazole due to hyperprolactinemia. Within 10 days of starting oral aripiprazole at a dose of $10 \mathrm{mg}$, the patient reported symptoms of double vision and blurring of vision in both eyes. On ophthalmologic evaluation, the patient did not have any refractory error. She did not have signs of any extrapyramidal symptoms, and preliminary neurological examination did not reveal any other abnormalities. Keeping in mind the possibility of the rare side effect of diplopia, she was advised to discontinue aripiprazole. On stopping the drug, the patient reported reduction in symptoms within 5 days with complete resolution in 10 days of stopping aripiprazole. The acute onset of diplopia and resolution of symptoms after stopping the drug correlates strongly with aripiprazole-induced diplopia. Till date, only two such cases were reported. Conclusion: Both the treating psychiatrists and ophthalmologists should be well aware that though rare, diplopia is a possible adverse effect of aripiprazole. Prompt discontinuation can reverse the condition.
\end{abstract}

Keywords: Adverse effects, Aripiprazole, Diplopia.

The Journal of Medical Sciences (2019): 10.5005/jp-journals-10045-00122

\section{BACKGROUND}

Aripiprazole is a second-generation antipsychotic (SGA) drug. Its primary mechanism of action comprises partial agonist activity at dopamine recptor (D2) and serotonin receptor (5HT1A) and antagonist activity at $5 \mathrm{HT} 2 \mathrm{~A}$ receptors. ${ }^{1}$ The drug has found widespread use in psychiatry, especially in schizophrenia and schizoaffective disorder, bipolar disorder, depression, and obsessive compulsive disorder (OCD). What sets it apart from other SGAs is its D2 partial agonist, which translates into an agent that acts as a functional antagonist under hyperdopaminergic conditions and an agonist under hypodopaminergic conditions.

Ocular side effects are the rarest to be reported, with only eight cases of myopia and two cases of diplopia related to aripiprazole being reported in literature till date. The drug has also been reported to have fewer adverse effects, such as extrapyramidal symptoms, sedation, QTc prolongation, and hyperprolactinemia. Commonly reported side effects with aripiprazole treatment are headache, nausea, constipation, somnolence, or insomnia. ${ }^{2}$ Here we report a case of aripiprazole-induced diplopia in a 28-year-old woman treated for depression with psychotic symptoms.

\section{Case Description}

A 28-year-old married woman with a 4-year-old child was brought to the outpatient department with a history of reduced activity, responsiveness, and talking for the last 1 month. She was not engaging in the daily activities that she previously used to do and would spend most of her time gazing into space. While she was very talkative previously, she stopped communicating verbally and only responded to questions by nodding or gesturing. A history of interpersonal relationship issues was reported with her husband and mother-in-law 2 months before the onset of the presenting complaints, and her husband later confided that she appeared sad

\begin{abstract}
${ }^{1-4}$ Department of Psychiatry, RajaRajeswari Medical College and Hospital, Bengaluru, Karnataka, India

Corresponding Author: Manaswini Mullapudi, Department of Psychiatry, RajaRajeswari Medical College and Hospital, Bengaluru, Karnataka, India, Phone: +918639915482, e-mail: dr.mullapudi22@ gmail.com

How to cite this article: Mullapudi M, Madan R, Vardhan V, et al. Aripiprazole-induced Diplopia: A Rare Case Report. J Med Sci 2019;5(3):73-74.
\end{abstract}

Source of support: Nil

Conflict of interest: None

and low at times. She had no medical comorbidities and her husband did not report any history of substance use or previous medications. On examination, she was cooperative and appeared depressed. She had hypoactivity, a staring look, and mutism. Physical examination did not reveal any abnormalities. Routine investigations such as complete blood counts, liver and renal function tests, and thyroid function tests were within normal limits and the patient had a body mass index (BMI) of 26.4 on initial assessment.

A working diagnosis of depression with catatonic symptoms was made (Bush-Francis catatonia rating scale score of 6).

The patient was initially started on oral escitalopram $10 \mathrm{mg}$ and oral risperidone $2 \mathrm{mg}$. However, on assessment after 3 weeks, she showed no improvement in symptoms with a score of 6 on BushFrancis catatonia rating scale. Risperidone dose was increased to $3 \mathrm{mg}$. She showed full improvement (Bush-Francis catatonia rating scale score of 0 ) within 2 months of initiation of treatment and was doing well on a dose of $3 \mathrm{mg}$ risperidone. However, 7 months later, the patient started complaining of galactorrhea. She also had concerns about her weight gain, as she had gained $9 \mathrm{~kg}$ in 7 months (BMI: 30.4). 
Therefore, after discussion with the patient, risperidone was tapered and then stopped and $10 \mathrm{mg}$ aripiprazole was started.

After 10 days of initiation of aripiprazole treatment, she started complaining of blurred and double vision in both eyes. An ophthalmologist opinion was taken, which revealed a vision of $6 / 6$ in both eyes, with an intraocular pressure (IOP) of 14 and $15 \mathrm{~mm} \mathrm{Hg}$ in right and left eyes, respectively. As the patient had no signs of extrapyramidal symptoms, diplopia induced by aripiprazole was suspected and the drug was stopped and patient was switched to $25 \mathrm{mg}$ of oral quetiapine. Patient reported improvement in symptoms of diplopia within 5 days of stopping the drug, with full resolution in 10 days.

\section{Discussion}

Aripiprazole was first synthesized in 1988 at the Otsuka Pharma Lab in Tokyo, Japan. It differs from other SGAs due to its partial agonist activity at D2 and 5HT2A receptors. It is a highly lipidsoluble quinolone derivative and its chemical structure does not resemble any of the available antipsychotics. Among the atypical antipsychotics, aripiprazole displays the lowest affinity for $a_{1}$-adrenergic, histamine, and muscarinic receptors. It is the first antipsychotic medication to receive the Food and Drug Administration (FDA) approval for adjunctive treatment of patients with major depressive disorder. Currently, aripiprazole is used in the treatment of schizophrenia and schizoaffective disorder, bipolar disorder, depression, and OCD. In general, aripiprazole seems to be a well-tolerated drug, especially with regard to metabolic side effects. The most commonly reported side effects include restlessness/akathisia, somnolence, and nausea. ${ }^{2}$ It also indicated a low incidence of weight gain and a low liability for inducing movement disorders. ${ }^{3}$ Other early data suggest that aripiprazole may induce reductions in plasma prolactin ${ }^{4}$ as well as in plasma glucose and lipid profiles. Ocular side effects from aripiprazole are very rare; and till date, eight cases of transient myopia related to aripiprazole were reported ${ }^{5}$ and only two previously reported cases of aripiprazole-induced diplopia. The first case was reported by Selvi et al. ${ }^{6}$ and another case of a 24 -year-old with schizophreniform disorder who reported symptoms of diplopia within 1 week of initiating $15 \mathrm{mg}$ oral aripiprazole. ${ }^{7}$ Ocular effects do not seem to be dose dependent, since the patients previously described in literature were medicated with different dosages, ranging from 3 to $20 \mathrm{mg} /$ day. The onset of symptoms was within a month, from 3 to 30 days, and the resolution after discontinuation was reported to be from 3 to 14 days.

\section{Conclusion}

Aripiprazole is largely viewed as a safer drug in comparison to other SGAs. This led psychiatrists to overlook ocular symptoms as side effects related to the drug. Therefore, the treating psychiatrists, physicians, and ophthalmologists should be aware of the possibility of ocular side effects and the fact that complete resolution occurs on discontinuation of the drug. Patients should also be warned about the possible visual symptoms that can occur, so that they immediately bring it to the attention of the treating doctor. This knowledge will help in early detection of adverse effects and immediate management.

\section{References}

1. Taylor DM. Aripiprazole: a review of its pharmacology and clinical use. Int J Clin Pract 2003;57(1):49-54.

2. Fleischhacker WW. Aripiprazole. Expert Opin Pharmacother 2005;6(12):2091-2101. DOI: 10.1517/14656566.6.12.2091.

3. Goodnick PJ, Jerry JM. Aripiprazole: profile on efficacy and safety. Expert Opin Pharmacother 2002;3(12):1773-1781. DOI: 10.1517/14656566.3.12.1773.

4. Gupta S, Lakshmanan DAM, Khastgir U, et al. Management of antipsychotic-induced hyperprolactinaemia. B J Psych Adv 2017;23(4):278-286. DOI: 10.1192/apt.bp.115.014928.

5. Abreu T, Pinheiro J. Aripiprazole induced myopia-case report and literature review: Aripiprazole induced myopia. Clin Schizophr Relat Psychoses 2019. DOI: 10.3371/CSRP.TAJP.121218.

6. Selvi Y, Atli A, Aydin A, et al. Aripiprazole-related acute transient myopia and diplopia: a case report. J Clin Psychopharmacol 2011;31(2):249-250. DOI: 10.1097/JCP.0b013e3182103493.

7. Atli A, Selvi Y, Yildiz A, et al. Aripiprazole-induced diplopia: a case report. Klinik Psikofarmakol Bülteni 2013;23(4):353-356. DOI: 10.5455/ bcp. 20130303114826. 